\title{
Vemurafenib: a new treatment for BRAF-V600 mutated advanced melanoma
}

\author{
This article was published in the following Dove Press journal: \\ Cancer Management and Research \\ 7 August 2012 \\ Number of times this article has been viewed
}

\author{
Rosalie Fisher \\ James Larkin \\ Department of Medical Oncology, \\ Royal Marsden Hospital, London, \\ United Kingdom
}

\begin{abstract}
The BRAF inhibitor, vemurafenib, has demonstrated improved progression-free and overall survival compared with chemotherapy in a randomized trial, and represents a new standard of care in patients with advanced melanoma harboring a BRAF-V600 mutation. A BRAF-V600 mutation is identified in approximately half of patients with cutaneous melanoma, and is unequivocally a biomarker predictive of profound clinical benefit for these patients. However, acquired vemurafenib resistance is a major clinical challenge and therapy is not yet curative. A substantial body of translational research has been performed alongside clinical trials of vemurafenib, providing key insights into the molecular basis of response and resistance. This review summarizes the development of vemurafenib for the treatment of BRAF-V600 mutant melanoma and discusses how knowledge of critical signaling pathways will be applied for its optimal clinical use in future.
\end{abstract}

Keywords: vemurafenib, melanoma, BRAF-V600, BRIM-3

\section{Introduction}

Malignant melanoma is the most aggressive type of skin cancer. Melanoma was diagnosed in approximately 85,000 people globally in $2008,{ }^{1}$ and is in general confined to economically developed countries. In particular, there is a high incidence in countries with fair-skinned populations, such as Northern Europe, the US, Australia, New Zealand, and South Africa. Despite only accounting for around 5\% of new cancer diagnoses in adults in the US, ${ }^{2}$ melanoma is of epidemiological significance because its worldwide incidence and death rate has been rising for the last 30 years, and continues to do so, ${ }^{3}$ and it affects a high proportion of younger adults. ${ }^{4}$

While the majority of patients present with a primary cutaneous malignant melanoma and are cured by surgical resection alone, metastasis to regional lymph nodes or distant sites occurs in a proportion and is associated with poor long-term survival. ${ }^{5}$ Furthermore, in those with visceral metastatic disease, melanoma is usually rapidly fatal, with an average survival of less than one year, ${ }^{5}$ and is associated with much morbidity. Prognosis from melanoma is determined by traditional anatomical staging; the risk of relapse from a primary melanoma correlates with features such as tumor thickness, ulceration, and mitotic rate, and in advanced melanoma, worsened clinical outcomes are observed in those patients with visceral metastases and those with an elevated lactate dehydrogenase level, presumed to reflect a higher burden of metastatic disease. ${ }^{5}$ Similarly, performance status was also found to be a prognostic variable in patients with stage IV melanoma treated in clinical trials. ${ }^{6}$
Correspondence: James Larkin Royal Marsden Hospital, Fulham Road, London SW3 6JJ, United Kingdom Tel +l 2078082198

Fax +l 20781 I 8I03

Email james.larkin@rmh.nhs.uk 
Palliative systemic therapy is the basis of management for metastatic melanoma, and until very recently, a global standard was dacarbazine, an alkylating chemotherapy agent. However, metastatic melanoma is regarded as being insensitive to cytotoxic chemotherapy, as evidenced by response rates to dacarbazine in the order of $10 \%$ and no proven survival benefit. ${ }^{7-10}$ Immunotherapy, including cytokine and vaccine treatments, provides the only alternative to chemotherapy but does not benefit the majority of patients, although durable responses have been observed in a small proportion of patients treated with high-dose interleukin-2. ${ }^{11}$

The generally held view that metastatic melanoma is refractory to systemic treatments was dramatically altered in 2010, when positive Phase III clinical trial results were reported for two novel agents. ${ }^{12,13}$ Both the anti-cytotoxic $\mathrm{T}$ lymphocyte-associated antigen-4 antibody, ipilimumab, and the small molecule inhibitor of BRAF, vemurafenib (formerly referred to as PLX4032 and RG7204), were shown to improve overall survival in patients with advanced melanoma in randomized controlled trials. Thus, these agents represent new standards of treatment, and are the basis for two broad but possibly complementary research directions in this disease. This review focuses on the development of vemurafenib, how it has transformed clinical practice in BRAF-V600 mutant melanoma, and how its use may be optimized in the future in this setting. It should be noted that the label for vemurafenib differs between licensing authorities in the US and Europe, and this review will refer to vemurafenib as treatment for BRAF-V600 mutant melanoma in accordance with the licensing conditions of the European Medicines Agency.

\section{Preclinical development of vemurafenib}

The promise of molecularly targeted therapy for melanoma began with the discovery of somatic mutations in the $B R A F$ gene in $66 \%$ of melanoma cell lines. ${ }^{14}$ Other oncogenes, such as NRAS, KIT, GNAQ, and GNA11, were found to characterize distinct clinicopathological subtypes of melanoma (Table 1). ${ }^{15-18}$ The shift from an anatomical to a molecular classification of melanoma has had profound implications for systemic drug therapy in advanced melanoma, and vemurafenib provides an example of how such an approach may lead to personalized treatment in this disease.

BRAF is an integral part of the RAS-RAF-MEK-ERK (mitogen-activated protein kinase) signal transduction pathway, a protein kinase cascade which regulates cellular growth, proliferation, differentiation, and survival in response to extracellular signals, including growth factors, cytokines, and hormones. ${ }^{19,20}$ This highly conserved pathway is strongly associated with human cancers; $R A S$ mutations are found in approximately $15 \%$ of human cancers, including melanoma, ${ }^{14,15,21-23}$ and $R A S$ and $B R A F$ mutations can occur in the same tumor types in a mutually exclusive pattern. The most common mutation in $B R A F$ is caused by a single amino acid substitution of valine for glutamine at codon 600 , representing the majority of $B R A F$ mutations found in human cancer. ${ }^{20}$ At least 40 missense mutations have been identified in $B R A F$, with the majority clustering in the kinase domain and causing constitutive activation. ${ }^{24}$ Determination of the crystal structure of mutant $B R A F$ revealed that most mutations involve amino acids which stabilize the interaction between the glycine-rich loop and the activation segment, leading to an inactive conformation; disruption of this interaction leads to the protein being held in the active state and elevating the kinase activity by 500 -fold. ${ }^{25,26}$ The potency of $B R A F$ as an oncogene and therefore its appeal as a therapeutic target was confirmed in further studies, all of which showed mutant $B R A F$ to be the prime activator of MEK-ERK signaling in cancer cells, independently of RAS, resulting in induction of proliferation and protection from apoptosis..$^{14,20,25-28}$ Several $B R A F$ mutants possess low kinase activity, but are able to activate MEK indirectly through recruitment of CRAF. ${ }^{25,26} \mathrm{~A}$ mouse model of BRAF-V600E mutant melanoma, in which tumors bore close pathological and genetic similarity to those seen in humans, established this mutant as a driving

Table I Molecular drivers in melanoma

\begin{tabular}{llll}
\hline Anatomical site of origin & Somatic gene mutation & Approximate frequency (\%) & References \\
\hline Skin & & & 10 \\
With chronic sun damage & BRAF & 15 & 15 \\
& NRAS & 60 & 15 \\
Without chronic sun damage & BRAF & 20 \\
Acral surfaces & NRAS & $6-40$ & $16,17,72$ \\
Mucosal surfaces & KIT & $15-40$ & $16,17,72$ \\
Uveal & KIT & $22-45$ & 18 \\
& GNAQ & $30-60$ & \\
\hline
\end{tabular}


event in melanomagenesis. ${ }^{29}$ However, the observation that $B R A F-V 600 E$ is capable of inducing senescence in melanocytes,${ }^{30}$ consistent with the common finding of $B R A F$ mutations in benign nevi, ${ }^{31}$ and the latency for the development of melanoma, suggest that additional genetic alterations are involved.

Initial therapeutic strategies to inhibit oncogenic $B R A F$ used sorafenib, subsequently shown to target multiple tyrosine kinases and have limited anti-BRAF activity. ${ }^{32}$ This was borne out by clinical studies in which sorafenib failed to demonstrate a significant antitumor effect. ${ }^{33,34}$ In contrast, vemurafenib is a small molecule inhibitor of mutant $B R A F$ developed through a crystallographic approach by Plexxikon Inc, resulting in greater potency for $B R A F-V 600 E\left(\mathrm{IC}_{50} 31 \mathrm{nM} / \mathrm{L}\right.$, versus $100 \mathrm{nM} / \mathrm{L}$ for wild-type $B R A F) .{ }^{35}$ Vemurafenib was chosen over a similar compound (PLX4720) for further development because of favorable pharmacokinetic properties. ${ }^{35,36}$

In vivo models confirmed the cellular selectivity of vemurafenib; in melanoma cell lines and xenograft models with V600E mutant $B R A F$, including Colo829, LOX, and A375, vemurafenib rapidly suppressed ERK signaling and hence tumor cell proliferation and survival, but lacked activity or conferred a growth advantage in wild-type $B R A F$ melanoma cell lines. ${ }^{37-41}$ MEK and ERK phosphorylation was also inhibited in melanoma cell lines expressing other mutations at the V600 position, including BRAFV600D, BRAFV600R, and $B R A F V 600 K$. The effect of three doses of vemurafenib was examined in a LOX xenograft model; inhibition of tumor growth was observed at all three dose levels, but tumor regression and mouse survival were clearly altered in a dose-dependent manner. ${ }^{37}$

In summary, shortly after $B R A F$ was established as a driver oncogene in melanoma, the small molecule BRAF inhibitor, vemurafenib, exhibited potent antitumor efficacy in biochemical and in vivo assays. As a result, it was driven rapidly through a clinical drug development program. Importantly, preclinical studies provided a foundation for research into the molecular mechanisms underlying particular clinical issues relevant to vemurafenib treatment, such as drug resistance and toxicity, which will be discussed later in this review.

\section{Clinical trials of vemurafenib}

Clinical trials of vemurafenib began in 2006. The BRAF Inhibitor in Melanoma (BRIM) Phase I, II, and III trials have now been completed, demonstrating striking consistency of clinical outcomes, and are summarized in Table 2.

\section{Phase I: BRIM-I}

This was a multicenter trial with a two-stage design; a dose-escalation phase, open to patients with any tumor type, and an extension phase, which included patients with $B R A F-V 600 E$ mutant melanoma only. ${ }^{42}$ The trial objectives were to establish the safety and pharmacokinetic profile of vemurafenib, and to make a preliminary assessment of clinical efficacy in those treated with the recommended Phase II dose of vemurafenib. Fifty-five patients (49 of whom had melanoma) and 32 patients were enrolled in each cohort, respectively. Eligibility for the extension phase was determined by a polymerase chain reaction (PCR) assay for the V600E $B R A F$ mutation in formalin-fixed, paraffin-embedded tumor tissue; this was not required for patients enrolled in the dose-finding phase, but in fact, a significant proportion of the cohort was identified as harboring the V600E mutation as the trial progressed. It should also be noted that the initial crystalline formulation of vemurafenib did not show any antitumor activity and was changed early in the trial to a microprecipitated bulk powder formulation, with substantially higher bioavailability.

A dose of $960 \mathrm{mg}$ twice daily was determined as the maximum tolerated and recommended Phase II dose. Side effects were mild to moderate in most patients and necessitated dose reduction in $41 \%$. Dose-limiting toxicities began at a dose of $720 \mathrm{mg}$ twice daily and most commonly involved arthralgia, fatigue, and cutaneous side effects, such as rash, photosensi-

Table 2 Summary of BRIM trial results

\begin{tabular}{|c|c|c|c|c|c|c|}
\hline Phase & Patient number & Patient population & Response rate (\%) & Median PFS (months) & Median OS (months) & References \\
\hline $\mathrm{I} / \mathrm{II}$ & $32^{\mathrm{a}}$ & $\begin{array}{l}\text { Advanced melanoma; } \\
\text { previous systemic therapy }\end{array}$ & $56^{b}$ & $>7$ & 12.6 & 42 \\
\hline II & 132 & $\begin{array}{l}\text { Advanced melanoma; } \\
\text { previous systemic therapy }\end{array}$ & 53 & 6.8 & 15.9 & 45 \\
\hline III & $\begin{array}{l}675 \\
\text { (336 treated with } \\
\text { vemurafenib) }\end{array}$ & $\begin{array}{l}\text { Advanced melanoma; } \\
\text { no prior systemic therapy }\end{array}$ & 48 & $\begin{array}{l}5.3^{c} \\
\text { HR } 0.26 \text { favoring } \\
\text { vemurafenib }\end{array}$ & $\begin{array}{l}\text { I3.2 } \\
\text { HR } 0.44 \text { favoring } \\
\text { vemurafenib }\end{array}$ & 13 \\
\hline
\end{tabular}

Notes: aWith BRAF mutations; 'by independent assessment; from the Summary of Product Characteristics, European Medicines Agency. Abbreviations: HR, hazards ratio; PFS, progression-free survival; OS, overall survival. 
tivity, cutaneous squamous cell carcinoma, and palmar-plantar dysesthesia. Squamous cell carcinoma was an unexpected finding, and occurred in $15 \%$ and $31 \%$ of the dose-escalation and extension cohorts, respectively. All but one of these were characteristic of the keratoacanthoma type, and none required intervention beyond simple excision.

A complete or partial tumor response occurred in 26 of 32 patients (81\%) who had melanoma with the V600E $B R A F$ mutation ( $56 \%$ by independent assessment) and 11 of 16 patients $(69 \%)$ with $B R A F-V 600 E$ mutant melanoma treated with doses of $240 \mathrm{mg}$ twice daily or higher in the dose-escalation phase. There was no evidence of tumor regression in five patients who had wild-type $B R A F$ metastatic melanoma. The rapidity of radiological and clinical responses observed in this study across all secondary tumor sites generated much excitement. Subsequently, the median overall survival in the 32 extension cohort patients was reported to be 12.6 months, and the 2-year survival rate was $35 \% .{ }^{43}$

The Phase I trial included two translational research components. Tumor biopsies at baseline and at day 15 were performed in seven patients in the extension cohort. Phosphorylated ERK, cyclin D1, and Ki-67, both indicators of mitogen-activated protein kinase pathway signaling and cell proliferation, were profoundly suppressed in these tumor samples in response to vemurafenib treatment. Recently, a functional imaging substudy was reported. ${ }^{44}$ All 31 patients in the extension cohort underwent baseline and day 15 imaging with fluorodeoxyglucose positron emission tomography, and for $100 \%$ of those who were treated at potentially therapeutic levels of vemurafenib $(n=27)$, at least a partial metabolic response was observed. This compares with $85 \%$ of patients in this cohort who achieved a tumor response in terms of conventional imaging and Response Evaluation Criteria In Solid Tumors (RECIST). Another notable feature of this substudy was the homogeneity of tumor metabolic response, as assessed by positron emission tomography, across tumor lesions, but a relationship between an early metabolic response and clinical outcomes, such as duration of response, progression-free survival, and overall survival, could not be demonstrated.

\section{Phase II: BRIM-2}

The Phase II trial of vemurafenib was again multicenter, but specifically tested the drug in patients with $B R A F-V 600$ mutant melanoma who had been previously treated (noting that approximately $50 \%$ of patients in BRIM-1 had received at least two prior lines of therapy). ${ }^{45} B R A F$ mutation status was assigned by the $\operatorname{cobas}^{\circledR}$ V600 PCR- based assay and verified by Sanger sequencing at a central laboratory. The main criterion for eligibility was progressive disease after at least one prior systemic therapy for advanced melanoma, and other criteria were standard, including the absence of active brain metastases.

The study recruited quickly and was over-enrolled; 132 patients were treated with vemurafenib, 122 of whom had BRAF-V600E mutations. The remaining 10 patients had $B R A F-V 600 K$ mutations. Approximately half had received one prior line of therapy, $27 \%$ had received two lines, and $22 \%$ had received three lines, usually chemotherapy. The majority of patients had advanced stage melanoma with either M1c disease or an elevated lactate dehydrogenase level. The primary endpoint of overall response rate was 53\% (95\% confidence interval [CI] 44-62) and responses were again rapid, with less than $15 \%$ of patients demonstrating disease progression at their first disease assessment. The median duration of response was 6.7 months. Four patients with $B R A F-V 600 \mathrm{~K}$ mutant melanoma had a partial response, and a further three had stable disease.

With a median follow-up of 12.9 months, median progression-free survival was 6.8 months (95\% CI 5.6-8.1) and median overall survival was 15.9 months (95\% CI 11.6-18.3). The latter was unchanged when those patients who had further treatment with ipilimumab in the follow-up period were excluded from the analysis. These results were viewed as highly encouraging, particularly in a challenging disease setting.

The toxicity profile in the Phase II study was very similar to that of the Phase I trial; $45 \%$ of patients had their dose reduced, and the most commonly reported side effects were arthralgia, rash, photosensitivity, fatigue, and alopecia. Cutaneous squamous cell carcinoma or keratoacanthoma developed in $26 \%$ of patients and a very small proportion were invasive cutaneous squamous cell carcinomas, but all were managed adequately with resection.

\section{Phase III: BRIM-3}

BRIM-3 was the registration trial for vemurafenib and randomized treatment-naïve patients with $B R A F-V 600 E$ mutant melanoma in a $1: 1$ ratio to vemurafenib or dacarbazine. ${ }^{13}$ Overall survival was the original primary endpoint of the study, but progression-free survival was added as a co-primary endpoint as a result of the efficacy seen in the Phase I and II clinical trials. Secondary endpoints included response and adverse event data.

Eligible patients had unresectable stage III or stage IV melanoma harboring the BRAF-V600E mutation, 
as determined by the cobas 4800 BRAF V600 mutation test. Six hundred and seventy-five patients were assigned to treatment during a 12-month period at multiple centers worldwide. The study population was characterized by features of advanced stage disease; stage M1c disease or an elevated lactate dehydrogenase was present in $60 \%-70 \%$ of patients. Nineteen patients had BRAF-V600K mutations and one had the V600D mutation. Treatments were administered at doses of $960 \mathrm{mg}$ orally twice daily for vemurafenib and $1000 \mathrm{mg} / \mathrm{m}^{2}$ intravenously every 3 weeks for dacarbazine.

Results of the interim analysis for BRIM-3, at a median follow-up of 3.8 months for patients in the vemurafenib arm and 2.3 months for those in the dacarbazine arm, were presented in the plenary session of the annual American Society of Clinical Oncology meeting in 2011, and revealed highly significant results in favor of vemurafenib. The hazards ratio for death in the vemurafenib group was 0.37 (95\% CI $0.26-0.55, P<0.001)$ and for progression-free survival in the vemurafenib group was 0.26 (95\% CI $0.20-0.33, P<0.001)$. Although most patients treated with vemurafenib had at least some tumor regression, an objective response occurred in $48 \%$ of vemurafenib patients ( $95 \%$ CI 42-55), compared with 5\% of patients treated with dacarbazine $(P<0.001)$. The response rate for dacarbazine was lower than predicted from earlier trials, and it is possible that $B R A F$ mutant metastatic melanomas are less sensitive to chemotherapy. However, $13 \%$ of patients in the dacarbazine group did not actually receive treatment, usually due to withdrawal of consent. Additionally, patients with elevated lactate dehydrogenase levels had been excluded from many previous trials of dacarbazine. Adverse events were consistent with those in earlier trials, and the incidence of cutaneous squamous cell carcinoma or keratoacanthoma or both was $18 \%$ in the vemurafenib group. Positive endpoints in this study were observed across all subgroups, and particularly in those patients deemed to have a poor prognosis on the basis of elevated lactate dehydrogenase or visceral disease burden.

On the basis of these results, the independent trial monitoring committee recommended that patients in the dacarbazine arm be allowed to cross over to receive vemurafenib. Updated results, with an additional 3 months of follow-up, were presented in $2011 .{ }^{43}$ The new hazards ratio for overall survival in the vemurafenib group was 0.44 (95\% CI $0.3-0.59)$ and the Kaplan-Meier estimate of median overall survival was 13.2 months compared with 9.6 months for dacarbazine patients. Six-month survival was estimated to be $83 \%$ and $63 \%$ for vemurafenib-treated and dacarbazinetreated patients, respectively. A global, multicenter safety trial of vemurafenib is currently recruiting. Vemurafenib has now received regulatory approval in the US and Europe, and in the latter is licensed as monotherapy for patients with advanced melanoma harboring a BRAF-V600 mutation.

\section{Vemurafenib resistance}

Clinical trial data indicate that almost all patients treated with vemurafenib for a $B R A F-V 600 E$ mutated melanoma experience some tumor shrinkage, even if it does not amount to a RECIST-defined response. However, it is also clear that most patients develop resistance to vemurafenib, manifested by progressive disease, and speed of relapse, once resistance is established, can be as rapid as the initial response to the drug. Additionally, there is a small proportion of patients whose tumors display primary refractoriness to vemurafenib.

In contrast with resistance to targeted therapies in other solid tumor types, acquired resistance to vemurafenib does not appear to result from secondary mutations in the $B R A F$ gene. Although resistance to vemurafenib in preclinical models could be conferred by the introduction of mutations into the "gate-keeper" region in the ATP-binding pocket of mutant $B R A F,{ }^{46}$ next-generation sequencing of 16 biopsy specimens with clinically acquired resistance to vemurafenib revealed retention of the original BRAF-V600E mutation and no secondary mutations ${ }^{47}$ This finding was confirmed in two further studies. ${ }^{48,49}$ The molecular mechanisms underlying vemurafenib are complex, but for simplicity can be divided into mitogen-activated protein kinase-dependent and mitogen-activated protein kinase-independent pathways.

\section{Mitogen-activated protein kinase- dependent mechanisms}

Clinical trial evidence for continued "addiction" of BRAF-V600 mutant melanoma cells to the oncogenic RASERK pathway comes from biopsies taken from progressing patients in the Phase I trial of vemurafenib. ${ }^{50}$ In 15 samples obtained at progressive disease, all had persistence of the original BRAF mutation, and in some cases ERK phosphorylation remained reduced.

There are three RAF proteins in cells (ARAF, BRAF, and CRAF) which under certain conditions form dimers. In cells with wild-type $B R A F$, inhibition of one molecule of the heterodimer (CRAF-BRAF) or homodimer (CRAF-CRAF) by a selective BRAF inhibitor results in transactivation of the drug-free protein and activation of the ERK pathway. ${ }^{51-53}$ In cells with the BRAF-V600 mutation and normal upstream $R A S$ levels, BRAF functions as a monomer; thus, when it is inhibited by a BRAF-V600 inhibitor, transactivation of 
heterodimers does not occur, and ERK signaling is suppressed. ${ }^{52}$ However, in the presence of upregulated $R A S$ (occurring through mutation in $R A S^{47}$ or an upstream activator of $R A S$ ), inhibition of V600-BRAF by a selective inhibitor releases it from its autoinhibited state in the cytosol and it is recruited to the plasma membrane where it dimerizes with CRAF, enhancing the activation of CRAF and allowing CRAF to hyperactivate the signaling pathway. ${ }^{51}$ Thus, inhibition of BRAF with a specific BRAF-V600 inhibitor appears to cause paradoxical CRAF-mediated activation of the ERK signaling pathway in wild-type $B R A F$ cells and in cells harboring both $B R A F-V 600 E$ and $R A S$ mutations; the former explains why selective $B R A F-V 600$ inhibitors such as vemurafenib only induce tumor regression in $B R A F-V 600$ mutant cells, and the latter may be partly responsible for drug resistance. It has also been shown that with chronic BRAF inhibition, BRAF-V600E cells acquire cross-resistance via flexible switching through the different RAS isoforms (ARAF and CRAF). ${ }^{48} \mathrm{~A}$ second mechanism to increase RAF dimer formation, independent of RAS, has been proposed. ${ }^{49}$ A splicing variant of BRAF-V600E, p61BRAF (V600E) has been identified, which lacks the exon coding region encompassing the RAS-binding domain and is associated with increased dimerization in cells with low levels of RAS. In cells expressing this variant, ERK signaling is resistant to vemurafenib, but sensitivity is restored with the introduction of a mutation which impairs dimerization, and BRAF splicing variants were found in tumors of six of 19 patients with acquired resistance to vemurafenib.

$B R A F-V 600 E$ copy number gain has been shown to exist in $20 \%$ of melanoma cell lines with acquired resistance to vemurafenib, ${ }^{54}$ and targeted massively parallel sequencing of a tumor from one patient with acquired resistance to vemurafenib identified an activating mutation in downstream kinase MEK $1 .{ }^{55}$ The $M E K 1^{\mathrm{C} 121 \mathrm{~S}}$ mutation was not present in the baseline biopsy sample, and conferred resistance to both RAF and MEK inhibition in vitro. This is the first report of a resistance mechanism involving an activating mutation downstream of the target kinase.

Mitogen-activated protein kinase pathway reactivation may also result from mechanisms which bypass RAF signaling. For example, Johannessen et al systematically introduced a library of DNA constructs, each encoding a different kinase, into $B R A F-V 600 E$ mutant melanoma cell lines, to identify candidates responsible for resistance to BRAF inhibition. ${ }^{56}$ Two kinases emerged as mediators of acquired resistance to PLX4720; CRAF, consistent with previous preclinical experiments $^{57}$ and mitogen-activated protein kinase 8 (MAP3K8, referred to as COT/TPL2), which functions upstream of MEK. Importantly, this study suggested that amplification of COT in melanoma cell lines may also be involved in de novo resistance.

\section{Mitogen-activated protein kinase- independent mechanisms}

In $B R A F-V 600 E$ mutant melanoma cell lines with acquired resistance to vemurafenib, gene expression signatures of persistent MEK-ERK activation were observed in one cell line, but two other cell lines retained vemurafenib-sensitive gene signatures. ${ }^{47}$ In the latter, platelet-derived growth factor $\beta$ upregulation was identified as an alternative survival pathway. The in vitro findings were confirmed in biopsies from clinical trial patients; platelet-derived growth factor $\beta$ overexpression and NRAS upregulation together accounted for acquired clinical resistance to PLX4032 in five of 12 study patients. Upregulation of insulin growth factor-1 receptor has also been found in resistant melanoma cells treated chronically with BRAF inhibitors, and enhanced activity of the IGF-1R-PI3K-Akt signaling pathway was evident in paired tissue samples (pretreatment and post-relapse) from one of five patients who had initially responded to vemurafenib. ${ }^{48}$

In summary, a body of evidence indicates that the mechanisms of resistance to vemurafenib are multiple and varied. Clinical resistance to therapy is arguably the biggest challenge facing oncologists treating BRAF-V600 mutant melanoma, and further insight into the molecular basis for resistance will likely change the way that vemurafenib is used in the clinic in future to optimize treatment outcomes.

\section{Other clinical challenges}

\section{Brain metastases}

The case of a 16-year-old patient with BRAF V600E metastatic melanoma with multiple brain metastases treated with vemurafenib has been reported. ${ }^{58}$ Symptomatic benefit was evident after one month of vemurafenib, and a partial radiological response in the brain was confirmed at 6 months. Preliminary results from an open-label, single-arm trial of vemurafenib in patients with brain metastases (pretreated by radiotherapy and/or chemotherapy) have also indicated that it is active in this context, with two of four patients showing some degree of response. ${ }^{59}$ These early results are highly significant because brain metastases from melanoma are very common, ${ }^{60}$ and particularly when viewed in the context of historical therapy for this clinical situation. Whole brain radiotherapy is considered standard but has not been shown to improve survival or to be associated with substantial morbid- 
ity, ${ }^{61}$ and the oral alkylating agent temozolomide crosses the blood-brain barrier but has very limited efficacy. ${ }^{62}$

\section{Vemurafenib in non-V600E mutant melanoma}

The BRAF-V600K mutation is the most common after $B R A F-V 600 E$, and has been noted to account for up to $30 \%$ of $B R A F$ mutations in melanomas in case series. ${ }^{63,64}$ Melanoma cell lines harboring the V600K mutation demonstrated reduced ERK signaling in response to PLX4032 treatment. ${ }^{40}$ There was an early report of a profound clinical response to vemurafenib in a patient with $B R A F-V 600 \mathrm{~K}$ mutant melanoma ${ }^{64}$ and a $40 \%$ response rate was observed in patients with the $\mathrm{V} 600 \mathrm{~K}$ variant in both the Phase II and III trials. ${ }^{13,45}$

It appears then that vemurafenib has in vitro and in vivo activity in non-V600E mutant melanoma, raising issues for companion diagnostic tests and drug licensing. The US Food and Drug Administration has issued guidance that "personalized" drugs will only be reviewed in parallel with the diagnostic test that was used to select patients for the drug in its clinical trials, ${ }^{65}$ and thus the licensed indication for vemurafenib in the US is for patients with $B R A F-V 600 E$ mutant advanced melanoma as defined by the Roche cobas assay. Although the number of $\mathrm{V} 600 \mathrm{~K}$ patients treated in clinical trials is small and there are no definitive survival data on this group to date, treatment with vemurafenib would seem reasonable and indeed the European label allows for this situation. In the US, the acceptability of treatment outside the drug's indication is debated, and may have consequences for reimbursement of treatment. This issue also has implications for the future of laboratory-developed tests in individual scientific institutions. ${ }^{65}$

\section{Secondary non-melanoma skin tumors}

Vemurafenib treatment is associated with a distinct dermatological toxicity profile, which includes a keratosis pilarislike rash, palmar-plantar dysesthesia, papillomas, and/or cutaneous squamous cell carcinomas. There appears to be a pattern of keratoacanthoma and squamous cell carcinoma development particular to vemurafenib treatment. The lesions are usually well differentiated and clinically indolent, occur early in the course of vemurafenib treatment (within the first 3 months) and are observed at higher frequencies in patients with ultraviolet exposure. Up to one third of patients treated in the three clinical trials of vemurafenib developed squamous cell carcinomas, and although all were managed adequately with excision, this gives cause for concern. Specifically, the risk of cutaneous and non-cutaneous squamous cell carcinomas has implications for the potential role of vemurafenib in an adjuvant setting.

Two studies confirm that the mutational profile of keratoacanthoma and squamous cell carcinomas associated with vemurafenib treatment is also unique, with frequent $R A S$ mutations, and support paradoxical activation of the mitogen-activated protein kinase pathway as the molecular basis for tumor development. ${ }^{66,67}$ The first of these analyzed 237 keratoacanthoma or squamous cell carcinoma tumor samples from patients treated with either vemurafenib or sorafenib and compared them with squamous cell carcinomas which developed spontaneously or because of immunosuppressive therapy. ${ }^{66} \mathrm{~A}$ higher rate of $R A S$ mutations, most commonly in $H R A S$, was found in patients treated with the RAF-inhibitor (21.1\% versus 3.2\%,P<0.01), and HRAS mutations were identified in $30 \%$ of samples from patients treated with vemurafenib. In the second study, Su et al identified $R A S$ mutations in $60 \%$ of keratoacanthoma and squamous cell carcinoma samples from vemurafenib-treated patients. ${ }^{67}$ Cell lines harboring the most prevalent mutation, HRAS Q6IL, showed evidence of ERK signaling despite treatment with PLX4720, and PLX4720 accelerated growth of skin tumors in an HRAS Q6IL mutant mouse model. Importantly, this model also demonstrated that concomitant treatment with a MEK inhibitor suppressed tumor development. Sorafenib, a pan-RAF inhibitor, has also been shown to induce keratinocyte proliferation in in vitro and in vivo. ${ }^{68}$ The proposed mechanism common to all three studies is that treatment with a BRAF inhibitor accelerates cutaneous tumor development in skin cells harboring pre-existing $(H) R A S$ mutations, by causing hyperproliferation through mitogen-activated protein kinase pathway signaling as in the models of vemurafenib resistance. It is also theorized that the life expectancy of most patients with metastatic melanoma is not long enough to allow development of non-cutaneous squamous cell carcinoma. However, the situation may be quite different if vemurafenib is used as adjuvant treatment, and one author has proposed prospective determination of $R A S$ status in all patients treated with BRAF inhibitors. ${ }^{69}$

\section{Future directions}

The unprecedented success of vemurafenib in the treatment of advanced melanoma has predictably led to proposals that it is trialed as treatment of earlier-stage disease, after surgical resection. Although there is a clear molecular basis for patient selection with the BRAF-V600 mutation, 
which predicts highly effective treatment, the issues of drug resistance and toxicity described above present challenges to curative therapy. Vemurafenib is currently being tested in other clinical settings, such as in patients with poor performance status and in those with non-V600E mutant melanoma (NCT01474551 and NCT01586195, respectively), and a study in pediatric patients is planned (NCT015193232).

The role of mitogen-activated protein kinase pathway restoration in resistance to vemurafenib and the development of cutaneous squamous cell carcinoma provides the rationale for combination therapy with a BRAF and a downstream MEK inhibitor. Another selective BRAF inhibitor, dabrafenib (GSK21118436), has been trialed with a MEK inhibitor, trametinib (GSK1120212) in a Phase I/II trial, with preliminary evidence of antitumor efficacy and safety. ${ }^{70}$ Notably, the rate of skin rash was substantially lower than that observed in the trials of vemurafenib, and no patient in this cohort to date has developed a squamous cell carcinoma. Combination strategies, such as vemurafenib and an IGF-1R inhibitor or an Akt inhibitor may also provide a means by which to overcome clinical resistance.

A trial of vemurafenib combined with the novel immunotherapy agent ipilimumab is already underway (NCT01400451). Oncogenic BRAF may have a role in immune evasion, and in a preclinical study, use of vemurafenib resulted in increased expression of melanocyte differentiation antigens and improved recognition by antigenspecific T lymphocytes, with no apparent impairment of T cell function. ${ }^{71}$

\section{Conclusion}

Vemurafenib is a new standard of care for patients with BRAF-V600 mutant melanoma, and the overall survival benefit established by the BRIM-3 trial represents a landmark event for the treatment of advanced melanoma. Importantly, all three clinical trials of vemurafenib demonstrated a high clinical benefit rate with manageable toxicity, and early evidence of vemurafenib activity in challenging clinical situations, such as the presence of brain metastases, suggests it has major therapeutic potential. A substantial body of translational research has been undertaken in parallel with clinical trials and already much is known of the complex molecular mechanisms underlying drug resistance and toxicity. However, it is clear that vemurafenib treatment is not yet curative, but can be optimized to improve patient outcomes even further.

\section{Disclosure}

JL has received honoraria from and served on advisory boards for GlaxoSmithKline and Bristol Myers Squibb. RF declares no conflict of interest.

\section{References}

1. Jemal A, Bray F, Center MM, et al. Global cancer statistics. CA Cancer J Clin. 2011;61:69-90.

2. Jemal A, Siegel R, Xu J, et al. Cancer statistics, 2010. CA Cancer J Clin. 2010;60:277-300.

3. World Health Organization. Cancer. Available from: http://www.who. int/cancer/. Accessed June 25, 2012.

4. Cancer Research UK: Cancer Statistics 2011. Available at http://info. cancerresearchuk.org/cancerstats/.

5. Balch CM, Gershenwald JE, Soong SJ, et al. Final version of 2009 AJCC melanoma staging and classification. J Clin Oncol. 2009;27:6199-6206.

6. Korn EL, Liu PY, Lee SJ, et al. Meta-analysis of Phase II cooperative group trials in metastatic stage IV melanoma to determine progressionfree and overall survival benchmarks for future Phase II trials. J Clin Oncol. 2008;26:527-534.

7. Chapman PB, Einhorn LH, Meyers ML, et al. Phase III multicenter randomized trial of the Dartmouth regimen versus dacarbazine in patients with metastatic melanoma. J Clin Oncol. 1999;17:2745-2751.

8. Middleton MR, Grob JJ, Aaronson N, et al. Randomized Phase III study of temozolomide versus dacarbazine in the treatment of patients with advanced metastatic malignant melanoma. J Clin Oncol. 2000;18:158-166.

9. Avril MF, Aamdal S, Grob JJ, et al. Fotemustine compared with dacarbazine in patients with disseminated malignant melanoma: a Phase III study. J Clin Oncol. 2004;22:111-1125.

10. Bedikian AY, Millward M, Pehamberger H, et al. Bcl-2 antisense (oblimersen sodium) plus dacarbazine in patients with advanced melanoma: the Oblimersen Melanoma Study Group. J Clin Oncol. 2006;24:4738-4745.

11. Atkins MB, Lotze MT, Dutcher JP, et al. High-dose recombinant interleukin 2 therapy for patients with metastatic melanoma: analysis of 270 patients treated between 1985 and 1993. J Clin Oncol. 1999;17: 2105-2116.

12. Hodi FS, O'Day SJ, McDermott DF, et al. Improved survival with ipilimumab in patients with metastatic melanoma. $N$ Engl $J$ Med. 2010;363:711-723.

13. Chapman PB, Hauschild A, Robert C, et al. Improved survival with vemurafenib in melanoma with BRAF V600E mutation. $N$ Engl J Med. 2011;364:2507-2516.

14. Davies H, Bignell GR, Cox C, et al. Mutations of the BRAF gene in human cancer. Nature. 2002;417:949-954.

15. Curtin JA, Fridlyand J, Kageshita T, et al. Distinct sets of genetic alterations in melanoma. $N$ Engl J Med. 2005;353:2135-2147.

16. Curtin JA, Busam K, Pinkel D, et al. Somatic activation of KIT in distinct subtypes of melanoma. J Clin Oncol. 2006;24:4340-4346.

17. Beadling C, Jacobson-Dunlop E, Hodi FS, et al. KIT gene mutations and copy number in melanoma subtypes. Clin Cancer Res. 2008;14: 6821-6828.

18. Van Raamsdonk CD, Griewank KG, Crosby MB, et al. Mutations in GNA11 in uveal melanoma. N Engl J Med. 2010;363:2191-2199.

19. Robinson MJ, Cobb MH. Mitogen-activated protein kinase pathways. Curr Opin Cell Biol. 1997;9:180-186.

20. Wellbrock C, Karasarides M, Marais R. The RAF proteins take centre stage. Nat Rev Mol Cell Biol. 2004;5:875-885.

21. Ball NJ, Yohn JJ, Morelli JG, et al. Ras mutations in human melanoma: a marker of malignant progression. J Invest Dermatol. 1994;102: 285-290.

22. van 't Veer LJ, Burgering BM, Versteeg R, et al. N-ras mutations in human cutaneous melanoma from sun-exposed body sites. Mol Cell Biol. 1989;9:3114-3116. 
23. Malumbres M, Barbacid M. RAS oncogenes: the first 30 years. Nat Rev Cancer. 2003;3:459-465.

24. Garnett MJ, Marais R. Guilty as charged: B-RAF is a human oncogene. Cancer Cell. 2004;6:313-319.

25. Wan PT, Garnett MJ, Roe SM, et al. Mechanism of activation of the RAF-ERK signaling pathway by oncogenic mutations of B-RAF. Cell. 2004;116:855-867.

26. Garnett MJ, Rana S, Paterson H, et al. Wild-type and mutant B-RAF activate C-RAF through distinct mechanisms involving heterodimerization. Mol Cell. 2005;20:963-969.

27. Wellbrock C, Ogilvie L, Hedley D, et al. V599EB-RAF is an oncogene in melanocytes. Cancer Res. 2004;64:2338-2342.

28. Karasarides M, Chiloeches A, Hayward R, et al. B-RAF is a therapeutic target in melanoma. Oncogene. 2004;23:6292-6298.

29. Dhomen N, Reis-Filho JS, da Rocha Dias S, et al. Oncogenic BRAF induces melanocyte senescence and melanoma in mice. Cancer Cell. 2009;15:294-303.

30. Michaloglou C, Vredeveld LC, Soengas MS, et al. BRAF E600associated senescence-like cell cycle arrest of human naevi. Nature. 2005;436:720-724.

31. Pollock PM, Harper UL, Hansen KS, et al. High frequency of BRAF mutations in nevi. Nat Genet. 2003;33:19-20.

32. Wilhelm SM, Carter C, Tang L, et al. BAY 43-9006 exhibits broad spectrum oral antitumor activity and targets the RAF/MEK/ERK pathway and receptor tyrosine kinases involved in tumor progression and angiogenesis. Cancer Res. 2004;64:7099-7109.

33. Eisen T, Ahmad T, Flaherty KT, et al. Sorafenib in advanced melanoma: a Phase II randomised discontinuation trial analysis. Br J Cancer. 2006; 95:581-586.

34. Hauschild A, Agarwala SS, Trefzer U, et al. Results of a Phase III, randomized, placebo-controlled study of sorafenib in combination with carboplatin and paclitaxel as second-line treatment in patients with unresectable stage III or stage IV melanoma. J Clin Oncol. 2009;27:2823-2830.

35. Bollag G, Hirth P, Tsai J, et al. Clinical efficacy of a RAF inhibitor needs broad target blockade in BRAF-mutant melanoma. Nature. 2010;467:596-599.

36. Tsai J, Lee JT, Wang W, et al. Discovery of a selective inhibitor of oncogenic B-Raf kinase with potent antimelanoma activity. Proc Natl Acad Sci U SA. 2008;105:3041-3046.

37. Yang H, Higgins B, Kolinsky K, et al. RG7204 (PLX4032), a selective BRAFV600E inhibitor, displays potent antitumor activity in preclinical melanoma models. Cancer Res. 2010;70:5518-5527.

38. Sala E, Mologni L, Truffa S, et al. BRAF silencing by short hairpin RNA or chemical blockade by PLX4032 leads to different responses in melanoma and thyroid carcinoma cells. Mol Cancer Res. 2008;6: 751-759.

39. Joseph EW, Pratilas CA, Poulikakos PI, et al. The RAF inhibitor PLX4032 inhibits ERK signaling and tumor cell proliferation in a V600E BRAF-selective manner. Proc Natl Acad Sci U S A. 2010;107: 14903-14908.

40. Halaban R, Zhang W, Bacchiocchi A, et al. PLX4032, a selective BRAF(V600E) kinase inhibitor, activates the ERK pathway and enhances cell migration and proliferation of BRAF melanoma cells. Pigment Cell Melanoma Res. 2010;23:190-200.

41. Tap WD, Gong KW, Dering J, et al. Pharmacodynamic characterization of the efficacy signals due to selective BRAF inhibition with PLX4032 in malignant melanoma. Neoplasia. 2010;12: 637-649.

42. Flaherty KT, Puzanov I, Kim KB, et al. Inhibition of mutated, activated BRAF in metastatic melanoma. $N$ Engl J Med. 2010;363: 809-819.

43. McArthur G, Hauschild A, Robert C, et al. Vemurafenib improves overall survival compared to dacarbazine in advanced BRAFV600Emutated melanoma: Updated survival results from a Phase III randomised, open-label, multicentre trial. Eur J Cancer. 2011; $47: 14$.
44. McArthur GA, Puzanov I, Amaravadi R, et al. Marked, homogeneous, and early [18F]fluorodeoxyglucose-positron emission tomography responses to vemurafenib in BRAF-mutant advanced melanoma. J Clin Oncol. 2012;30:1628-1634.

45. Sosman JA, Kim KB, Schuchter L, et al. Survival in BRAF V600mutant advanced melanoma treated with vemurafenib. $N$ Engl J Med. 2012;366:707-714.

46. Whittaker S, Kirk R, Hayward R, et al. Gatekeeper mutations mediate resistance to BRAF-targeted therapies. Sci Transl Med. 2010;2: $35-41$.

47. Nazarian R, Shi H, Wang Q, et al. Melanomas acquire resistance to B-RAF(V600E) inhibition by RTK or N-RAS upregulation. Nature. 2010;468:973-977.

48. Villanueva J, Vultur A, Lee JT, et al. Acquired resistance to BRAF inhibitors mediated by a RAF kinase switch in melanoma can be overcome by cotargeting MEK and IGF-1R/PI3K. Cancer Cell. 2010;18:683-695

49. Poulikakos PI, PersaudY, Janakiraman M, et al. RAF inhibitor resistance is mediated by dimerization of aberrantly spliced BRAF(V600E). Nature. 2011;480:387-390.

50. McArthur GA, Ribas A, Chapman PB, et al. Molecular analyses from a Phase I trial of vemurafenib to study mechanism of action (MOA) and resistance in repeated biopsies from BRAF mutation-positive metastatic melanoma patients. J Clin Oncol. 2011;29:Abstr 8502.

51. Heidorn SJ, Milagre C, Whittaker S, et al. Kinase-dead BRAF and oncogenic RAS cooperate to drive tumor progression through CRAF. Cell. 2010;140:209-221.

52. Poulikakos PI, Zhang C, Bollag G, et al. RAF inhibitors transactivate RAF dimers and ERK signalling in cells with wild-type BRAF. Nature. 2010;464:427-430.

53. Hatzivassiliou G, Song K, Yen I, et al. RAF inhibitors prime wild-type RAF to activate the MAPK pathway and enhance growth. Nature. 2010;464:431-435.

54. Shi H, Moriceau G, Kong X, et al. Melanoma whole-exome sequencing identifies (V600E)B-RAF amplification-mediated acquired B-RAF inhibitor resistance. Nat Commun. 2010;3:724.

55. Wagle N, Emery C, Berger MF, et al. Dissecting therapeutic resistance to RAF inhibition in melanoma by tumor genomic profiling. $J$ Clin Oncol. 2011;29:3085-3096.

56. Johannessen CM, Boehm JS, Kim SY, et al. COT drives resistance to RAF inhibition through MAP kinase pathway reactivation. Nature. 2010;468:968-972.

57. Montagut C, Sharma SV, Shioda T, et al. Elevated CRAF as a potential mechanism of acquired resistance to BRAF inhibition in melanoma. Cancer Res. 2008;68:4853-4861.

58. Rochet NM, Kottschade LA, Markovic SN. Vemurafenib for melanoma metastases to the brain. N Engl J Med. 2011;365:2439-2441.

59. Dummer R, Rinderknecht J, Goldinger SM, et al. An open-label pilot study of vemurafenib in previously treated metastatic melanoma patients with brain metastases. J Clin Oncol. 2011;29:Abstr 8548.

60. Bafaloukos $\mathrm{D}$, Gogas $\mathrm{H}$. The treatment of brain metastases in melanoma patients. Cancer Treat Rev. 2004;30:515-520.

61. Fife KM, Colman MH, Stevens GN, et al. Determinants of outcome in melanoma patients with cerebral metastases. J Clin Oncol. 2004;22: 1293-1300.

62. Agarwala SS, Kirkwood JM, Gore M, et al. Temozolomide for the treatment of brain metastases associated with metastatic melanoma: a Phase II study. J Clin Oncol. 2004;22:2101-2107.

63. Long GV, Menzies AM, Nagrial AM, et al. Prognostic and clinicopathologic associations of oncogenic BRAF in metastatic melanoma. J Clin Oncol. 2011;29:1239-1246.

64. Rubinstein JC, Sznol M, Pavlick AC, et al. Incidence of the V600K mutation among melanoma patients with BRAF mutations, and potential therapeutic response to the specific BRAF inhibitor PLX4032.J Transl Med. 2010;8:67.

65. Schmidt C. Challenges ahead for companion diagnostics. J Natl Cancer Inst. 2012;104:14-15. 
66. Oberholzer PA, Kee D, Dziunycz P, et al. RAS mutations are associated with the development of cutaneous squamous cell tumors in patients treated with RAF inhibitors. J Clin Oncol. 2011;30:316-321.

67. Su F, Viros A, Milagre C, et al. RAS mutations in cutaneous squamouscell carcinomas in patients treated with BRAF inhibitors. NEngl JMed. 2012;366:207-215.

68. Arnault JP, Mateus C, Escudier B, et al. Skin tumors induced by sorafenib; paradoxic RAS-RAF pathway activation and oncogenic mutations of HRAS, TP53, and TGFBR1. Clin Cancer Res. 2011;18: $263-272$.

69. Weeraratna AT. RAF around the edges - the paradox of BRAF inhibitors. N Engl J Med. 2012;366:271-273.
70. Infante JR, Falchook GS, Lawrence DP, et al. Phase I/II study to assess safety, pharmacokinetics, and efficacy of the oral MEK $1 / 2$ inhibitor GSK1120212 (GSK212) dosed in combination with the oral BRAF inhibitor GSK2118436 (GSK436). J Clin Oncol. 2011;29:Abstr CRA8503.

71. Boni A, Cogdill AP, Dang P, et al. Selective BRAFV600E inhibition enhances T-cell recognition of melanoma without affecting lymphocyte function. Cancer Res. 2010;70:5213-5229.

72. Handolias D, Hamilton AL, Salemi R, et al. Clinical responses observed with imatinib or sorafenib in melanoma patients expressing mutations in KIT. Br J Cancer. 2010;102:1219-1223.

\section{Publish your work in this journal}

Cancer Management and Research is an international, peer-reviewed open access journal focusing on cancer research and the optimal use of preventative and integrated treatment interventions to achieve improved outcomes, enhanced survival and quality of life for the cancer patient. The journal welcomes original research, clinical \& epidemiological studies, reviews \& evaluations, guidelines, expert opinion \& commentary, case reports \& extended reports. The manuscript management system is completely online and includes a very quick and fair peerreview system, which is all easy to use. Visit http://www.dovepress.com/ testimonials.php to read real quotes from published authors. 